\title{
Quality control of delay-Doppler maps for stare processing
}

\author{
Giuseppe Grieco, Ad Stoffelen, Marcos Portabella, Maria Belmonte Rivas, Wenming Lin, Fran Fabra
}

\begin{abstract}
A quality control scheme for TechDemoSat 1 (TDS1) and CYclone GNSS (CYGNSS) delay-Doppler maps (DDMs) is presented and the results of its application to a dataset of more than 700,000 DDMs are discussed. This scheme is proven to be effective for such purpose and its output indices can be successfully used as quality indicators of the DDM. This study shows that most of the TDS-1 DDMs are affected by some distortions that are attributable to an insufficiently accurate estimation of the specular point location. The errors, moreover, can severely alter the symmetry of the iso-delay lines with respect to the iso-Doppler lines leading to an asymmetry in the arrival time of the waveforms. Furthermore, these errors may affect the convolution of the GNSS reflected signal with the Woodward Ambiguity Function, leading to an unwanted redistribution of the incoming echo energy among the DDM bins. Such distortions may in turn affect the accuracy of the wind field retrieval using either the stare processing approach or the more consolidated methods of inverting a Geophysical Model Function based on the DDM peak and/or leading edge slope.
\end{abstract}

Index Terms-GNSS-R, quality control, accuracy, error, wind, stare processing.

\section{INTRODUCTION}

A CCURATE sea surface wind field estimation and monitoring are important for a variety of oceanographic, atmospheric and climate applications. For example, the success of an off-shore wind farm installation for the production of renewable energy strictly relies on the capability of accurately assessing the wind climatology of a marine site. Also, accurate sea surface wind field estimation plays a key role in weather forecasts [1]. The wind field is recognized as one of the 54 Essential Climate Variables (ECV) by the World Meteorological Organization (WMO). WMO requires that the sea surface wind speed is retrieved with a bias of less than 0.5 $\mathrm{ms}^{-1}$ and standard deviation below $2 \mathrm{~ms}^{-1}$. Furthermore, it should be ideally retrieved with a frequency of 1 sample every 3 hours and with a spatial resolution better or equal to $10 \mathrm{~km}$ [2]. Stoffelen et al. demonstrated that the introduction of the OSCAT scatterometer, separated in time from ASCATA/B by 2,5 hours provided substantial complementary impact in Numerical Weather Prediction (NWP) applications [3]. At the moment, sea surface winds are routinely retrieved from the scatterometers ASCAT-A and B onboard the European Agency for the Exploitation of Meteorological Satellites (EUMETSAT) polar orbiting platforms MetOp A/B [4] and from Scatsat-1, onboard the Indian Space Research Organisation (ISRO). These scatterometer winds achieve accuracies $<1$ $m s^{-1}$, with a spatial resolution equal or higher than $25 \mathrm{~km}$ [5], in line with the WMO requirements. However, even if the multiple upcoming satellite scatterometer missions, i.e., the Chinese-French Oceanography Satellite (CFOSAT [6]), which will be jointly operated by the Chinese National Space Administration (CNSA) and by the French Space Agency (CNES), the OSCAT on board the Indian OceanSat3/A [7], the HSCAT on board the Chinese HY-2B/C [7], and the ASCAT$\mathrm{C}$ onboard the MetOp-C, the frequency requirement will be far from being matched in the following time intervals: 00:00 - 06:00 and 12:00 - 18:00 at Local Time Ascending Node (LTAN) [8]. All these ocean wind vector missions are planned for launch in 2018 or 2019. The complementarity of wind measurements plays a key role in weather forecasts. To depict processes, such as the diurnal cycle, ocean interaction or atmospheric mesoscale convection, additional measurements in the early morning and afternoon will be valuable [3].

Reflected Global Navigation Satellite System (GNSS-R) signals of opportunity could potentially be used in order to improve the temporal coverage of ocean wind measurements. This is one of the main objective of the EUMETSAT funded project GNSS-R Observation Operator Development and Impact Evaluation (GOODIE [9]) that is being carried out at the Royal Netherlands Meteorological Institute (KNMI) in collaboration with the Institute of Marine Science of the Spanish National Science Council (ICM-CSIC). Indeed, several studies have demonstrated the feasibility of retrieving the wind field from ocean reflected Global Positioning System (GPS) signals acquired by ground and airborne based receivers [10], balloons [11] and satellite [12]. The idea of exploiting GNSS signals for ocean wind retrievals dates back to the late 90s ( [13], [14]). [15] formulated the bistatic radar equation for L-band GNSS-R in the geometric optics limit. Recently, [16], [17] addressed the same problem in a more general form, where the geometric optics limit constraint has been removed.

GNSS constellations are designed in such a way that at least 4 satellites (usually more) are visible at the same temporal instance from every point on the globe, in order to accurately resolve the position of the receiver. At the moment, there are two ongoing satellite-based GNSS-R missions: a) the UK TechDemoSat-1 (TDS-1) [18] and b) the U.S. Cyclone GNSS (CYGNSS) [19]. The first one was launched in 2014 and consists of a single polar orbiting satellite carrying on board the Space GNSS Receiver Remote Sensing Instrument (SGR-ReSI) and is operated by Surrey Satellite Technology Limited (SSTL). The second mission, launched at the end of 2016, is operated by the National Aeronautics and Space Administration (NASA) and consists of 8 microsatellites that are equally spaced on an 35-degrees inclined orbit with respect to the equator. The choice of the orbit is due to the main aim of the mission, which is the monitoring of tropical cyclones. 
Both missions acquire delay-Doppler maps (DDMs) with a frequency of $1 \mathrm{~Hz}$. Therefore, a large quantity of GNSS-R signals of opportunity is available for analyzing the potential impact of GNSS-R wind data assimilation into NWP models. To the best of our knowledge, at the moment two approaches exist for ocean wind speed retrieval from satellite GNSS$\mathrm{R}$ echoes. The first one consists of inverting an empirical geophysical model function (GMF) relating the signal to noise ratio (SNR) [20], [21] or the normalized bistatic radar cross section (NBRCS) [12] of the DDM signal pertinent to the specular area to the unknown ocean parameters. The second approach consists of minimizing the variance of the wind speed retrieval by performing a weighted average of two different retrievals, i.e., those obtained by inverting the GMFs of two different observables [22]. A similar approach is followed by [23], but focusing on defining alternative observables to optimize wind retrieval quality. The approach developed by [22], in which the observables are the average NBRCS (DDMA) of the approximately $25 \mathrm{~km}$ square area around the specular point (SP) and the leading edge slope (LES) of the average waveform (WF) of the 5 Doppler channels in the interval $[-1,1] k H z$, is routinely used for wind speed retrievals from CYGNSS measurements. For CYGNSS, the uncertainty of the retrieved wind speed has been reported to be around $1.4 \mathrm{~ms}^{-1}$ in the wind speed range $0-20 \mathrm{~ms}^{-1}$, being lower in the range 0-7 $\mathrm{ms}^{-1}$ and progressively degrading with wind increasing speed [24]. For TDS-1, the reported overall uncertainty is around $2 \mathrm{~ms}^{-1}$. A more thorough and objective validation with more consolidated wind measurements is however needed to verify whether the mission requirements in terms of wind quality are actually met. In data assimilation, where the observation and NWP model information are combined, the best impact is achieved when the observation error variance is lower than the NWP model error variance. If the former is higher than the latter, it may in fact be difficult to demonstrate observation impact altogether. From [4], [25], we estimate that the NWP wind speed error variance on the scatterometer spatial scale is well below 3 $m^{2} s^{-2}$, while the scatterometer wind speed observation error variances are well below $1 \mathrm{~m}^{2} s^{-2}$. With GNSS-R system error variances of about $4 \mathrm{~m}^{2} \mathrm{~s}^{-2}$, it will be very difficult to extract the relevant geophysical processes and dynamics from the GNSS-R observations in NWP data assimilation. The reasons for a such poor accuracy at wind speed higher than $>7 \mathrm{~ms}^{-1}$ are various and can be summarized by the poor sensitivity of the GNSS-R observables to wind speed changes, the low SNR, and the significant measurement biases as further elaborated in this manuscript. Furthermore, the dependency of the various observables on other geophysical parameters such as the significant wave height $(\mathrm{SWH})$, the sea surface temperature (SST), etc. has not yet been properly assessed. Also note that, the noise coming from L-band anthropogenic sources [26] can severely degrade the GNSS-R signal.

A quite different approach is the so-called stare processing, used by [27] for the estimation of the mean square slope (mss). Such approach exploits the multiple looks of the same surface target on a series of $1 \mathrm{~Hz}$ DDMs over the ambiguity-free area of the DDM (commonly known as the "horseshoe").
This paper shows a stand-alone quality control scheme which aims at classifying the DDMs that are useful for the stare processing approach. Indeed, a substantial amount of DDMs suffer from unexpected features that can distort the horseshoe. Such DDMs cannot be properly used in stare processing because the DDM bins may not be representative of the wind conditions in the target area.

The paper is organized as follows: section II addresses the motivation for a quality control scheme; section III describes the dataset used in this work; section IV describes the rational of the scheme and its detailed implementation; section $\mathrm{V}$ shows the results of its application and, finally, in section VI the conclusions and some suggestions for the upcoming and future work can be found.

\section{Formulation OF THE PROBLEM}

The reflected GNSS signals are weak and distorted, therefore, the standard GNSS closed loop tracking techniques cannot be successfully applied [28]. As such, in order to compute the DDM on board the satellite platform, three specific algorithms are required: a) calculation of the SP location, b) calculation of the delay and Doppler estimates of the SP path, c) allocation of reflections to processing channels. The SP location is computed by means of the so-called QuasiSpherical (QS) approximation [28], [29]. It is important to stress that the accuracy of the SP calculation is crucial for the quality of the DDM because it can affect the algorithms in b) and c) mentioned above. TDS-1 data are only available at Level 1a data or higher. The Level 1a data is the result of on board post processing of Level 0 data, which prevents a reprocessing of the raw signal.

In order to apply the stare processing approach, it is not necessary, but of course recommended, to transform the Level $1 \mathrm{~b}$ data in its equivalent $\operatorname{NBRCS}\left(\sigma_{0}\right)$. Indeed, $\sigma_{0}$ is the physical quantity that depends on the geophysical parameters of interest, i.e., the mss and therefore, the wind speed [15]. This can be done by inverting the bistatic radar equation addressed in [17] (equation 13). Actually, this equation only accounts for the non-coherent part of the GNSS-R echo, the coherent part of which limits its effect to low wind regimes (up to $3 \mathrm{~ms}^{-1}$ ) and only affects the DDM bins surrounding the SP [17]. As the deconvolution of the signal is impossible, a useful way to address this problem is by using an approximation of equation 13 in [17], that reads [30]:

$$
P_{R} \approx \frac{\lambda^{2} P_{T} D_{T} D_{R} \sigma_{0} A}{(4 \pi)^{3} R_{0}^{2} R_{1}^{2} L_{0} L_{1}}
$$

In equation $1, \lambda$ is the wavelength of the GNSS carrier signal, $P_{R}$ and $P_{T}$ represent respectively the received and the transmitted power; $D_{R}$ and $D_{T}$ are, respectively, the transmitter and receiver antenna directivity factors; $R_{0}$ and $R_{1}$ are, respectively, the distance between the scatterer element and the transmitter and the receiver; and $L_{0}$ and $L_{1}$ are the atmospheric loss to and from the surface respectively. Finally, $A$ is the integral of the WAF, that represents the effective area that is being sounded and that depends on both the incidence angle and the satellite velocity vectors. Equation 1 is meant for 
every delay-Doppler bin $(\tau, f)$ of the DDM. Therefore, except $P_{T}$, which depends on the Pseudo Random Noise (PRN) code, all terms are strictly dependent on $(\tau, f)$. For the sake of clarity, the latter notation has been omitted from equation 1 . $P_{T}$ depends on the PRN code, but also shows a variability on time, which represents a large uncertainty factor [31] [32]. Equation 1 can be inverted in order to estimate $\sigma_{0}$, given that the user knows the transmitted power, both antenna directivity factors and the geometric factors $A, R_{0}, R_{1}$. The accuracy of the geometric factors depends on the accuracy of the bistatic configuration geometry, therefore on the positions and the velocities of both the receiver and the transmitter, and on the position of the SP, as said before.

The observation of unexpected features in most of the DDMs of this dataset led us to the development of the quality control scheme presented in this manuscript. In the following sections, it is shown that the main cause for such distortions is the inaccuracy of the SP position estimate. In figures 1a (1c), the arrival time of the waveform at $500 \mathrm{~Hz}(-500 \mathrm{~Hz})$ is anticipated with respect to the waveform at $0 \mathrm{~Hz}$. This unexpected feature indicates a Doppler shift of the DDM peak. Furthermore, these DDMs are not simply rigidly shifted. Indeed, the arrival times of the waveforms does not properly reflect the symmetry between the delay and Doppler iso-lines, giving the feeling of a "rotation" of the DDM horseshoe. This aspect will be clarified with some examples later on in the paper (figure $4 \mathrm{~d}$ of section V).

Shifts may also be present in the delay axis. One of such cases is depicted in figure 4, which will be discussed in section $\mathrm{V}$. These problems in the DDM can severely affect the $\sigma_{0}$ estimation, therefore, they should be flagged as poor quality.

\section{DATASET}

The TDS-1 Level $1 \mathrm{~b}$ data are in the form of DDM power counts. The necessary instructions for their radiometric calibration are available in [28]. In this paper, only uncalibrated data will be used and referred to as TDS-1 DDMs. However, it is important to stress that as the quality control scheme presented in this paper is based on correlation functions, the results are identical for both calibrated and uncalibrated data. Indeed, correlation functions are invariant for a linear transformation, and, as shown in the TDS-1 data manual [28], radiometric calibrated data are a linear combination of the uncalibrated power.

The dataset that has been used in this study consists of about 720,000 full TDS-1 Level 1b DDMs acquired in the period from the 4th of July 2015 until the 19th of June 2016 in fixed gain mode (FGM). Note that in comparison with the unmonitored automatic gain control (UAGC) mode (in which the SGR-ReSI operated during the first 9 months of the TDS-1 mission, i.e., until April 2015), the fixed gain mode (switched on in May 2015) is more suitable for calibration purposes since the receiver absolute power levels are known [21]. TDS1 DDMs are delivered on a Doppler grid spanning from -5 $k \mathrm{~Hz}$ to $4.5 \mathrm{kHz}$ with a grid spacing of $500 \mathrm{~Hz}$, and on a delay grid of 128 bins with a grid spacing of a quarter $\tau_{c}$. One chip corresponds to $0.977 \mu \mathrm{s}$, or, equivalently, to $293 \mathrm{~m}$. The
DDMs of this dataset have incidence angles that span from $0^{\circ}$ to $70^{\circ}$, all over the globe, from $-80^{\circ}$ to $80^{\circ}$ in latitude.

The DDMs have been collocated with the operational $3 \mathrm{~h}$ forecast winds from the European Centre for Medium-Range Weather Forecasts (ECMWF) NWP output. These winds are used as input for the computation of synthetic DDMs that are used in the scheme as described later in section IV. NWP winds have been temporarily and spatially interpolated to the TDS-1 DDMs. That is, 3-hourly ECMWF forecast winds on a regular grid of 0.5625 in latitude/longitude are interpolated to the TDS-1 SP location and time. In addition, the entire dataset has been collocated with ASCAT winds according to the following spatio-temporal constraints: a) the ASCAT acquisition time is within \pm 20 minutes with respect that of TDS-1; b) the distance between the TDS-1 SP and the ASCAT wind vector cell is within $25 \mathrm{~km}$.

WAVPY [33] is a quite flexible open source tool that has been designed for analysis and simulation of GNSS-R waveforms. In this work, WAVPY has been used to simulate the DDM features using collocated NWP wind field information for a consistency check with real TDS-1 DDMs. As an alternative, the user can provide mss as input. If only the wind is provided, mss is computed based on [34]. This choice has been adopted in this study. All the information related to the acquisition geometry (acquisition time, transmitter and receiver positions and velocity vectors) comes from TDS- 1 metadata, except for the SP location. This is computed directly by WAVPY according to the ellipsoid WGS-84 [35].

\section{Methodology}

In order to correctly assess both the delay/Doppler shifts and the distortions, two tests based on cross-correlation functions and on the use of simulated DDMs have been designed.

\section{A. Quality control test 1}

The first test $\left(Q T_{1}\right)$ aims at evaluating both delay and Doppler shifts. It is based on the cross correlation function between real and simulated DDMs. It is applied in the inner core of the DDM, defined as the region of the DDM between $-1.25 \tau_{c}$ and $6.75 \tau_{c}$ in delay and between $-0.5 k \mathrm{~Hz}$ and $0.5 \mathrm{kHz}$ in Doppler (for a total of $32 \times 3$ bins, red box of figure $2 \mathrm{a})$. Before applying the cross correlation, both the real and the simulated DDMs are normalized and reshaped according to the following procedure:

- a) Evaluation of the noise level;

- b) Subtraction of the noise level from the inner core of the DDM;

- c) Reshaping of the inner core in a 1-dimensional array (1D-DDM);

- d) Normalization of the 1-D array by its maximum value (figures $2 \mathrm{~b}, 2 \mathrm{c}$ );

The evaluation of the noise level of the real DDM is computed by averaging the DDM rows between 30 and 50 delay bins. It is well known that the part of the DDM below $-1 \tau_{c}$ is representative of an area above the Earth's surface, thus measuring the DDM noise [28]. The SP point is located at bin 64 in all the TDS-1 DDMs analyzed in this paper. As 


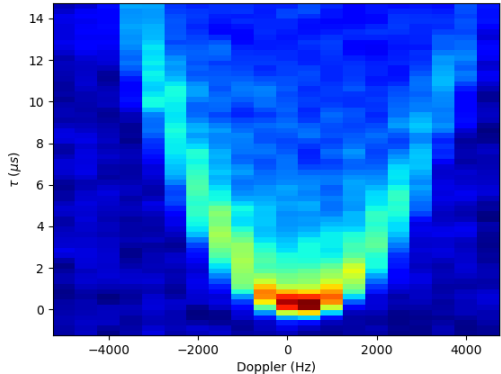

(a) Positive Doppler shift

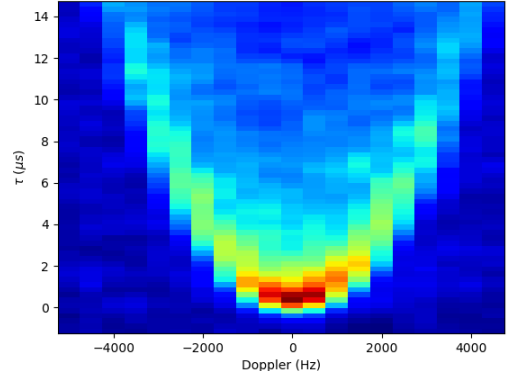

(b) Regular DDM

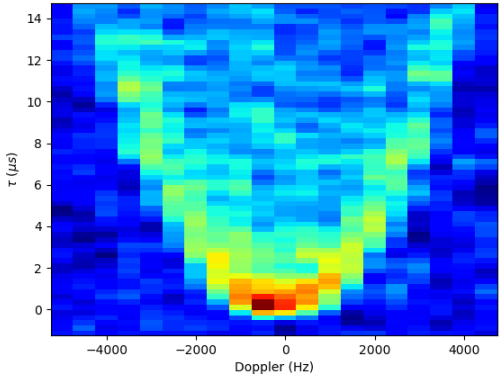

(c) Negative Doppler shift

Fig. 1. Examples of correct (1b) and incorrect position of the DDM peak (1a 1c)

such, the delay bins in the interval $[30,50]$ are well within the noise region of the DDM. The inner core of the real DDM is shifted bin-by-bin in delay and Doppler in the intervals \pm 5 and \pm 2 bins, respectively, for the delay and the Doppler axis. At each shift, the cross correlation coefficient between the mentioned 1D-DDMs is computed. Therefore, a matrix of $11 \times 5$ cross correlation values $(\mathbf{C})$ is obtained. The delay/Doppler lags corresponding to the maximum cross correlation value represent the delay/Doppler shifts. The perfectly aligned DDM should give the result $\arg \max _{i, j} \mathbf{C}=(6,3)$ where $i$ and $j$ are respectively the delay and Doppler indices, corresponding to null lags in both axes.

Figures $2 \mathrm{~b}$ and $2 \mathrm{c}$ show respectively a case in which the real and the simulated DDMs are perfectly aligned and a case in which they are not. In both figures, additional information on the best cross correlation coefficient $(\rho)$, the assessed values for both shifts in delay and Doppler ( $\Delta \mathrm{d}$ and $\Delta \mathrm{D}$ respectively) and the incidence angle at the specular point $(\theta)$, is provided. Cases similar to that depicted in fig. $2 \mathrm{c}$ are much more common than those in figure $2 \mathrm{~b}$. From figure $2 \mathrm{c}$ one can see several features: it is evident that there is a shift, with respect to the simulated inner core of the DDM; furthermore, the real DDM is noisy, giving rise to some secondary peaks, which are probably due to residual speckle noise and/or thermal noise (appendix III of CYGNSS Handbook [36]) or to contamination through the secondary lobes of the antenna gain pattern. Finally, it seems that the peak of the real DDM is misplaced with respect to the expected position. This could simply be due to noise. Indeed, it is evident that in some situations (2c), the noise is not negligible and this can mask the real DDM peak. It is evident that $\rho$ can be used as a quality indicator of the DDM. This aspect will be discussed in more details in section V.

\section{B. Quality control test 2}

The second test $\left(Q T_{2}\right)$ is designed to assess the presence of any distortion, which is perceived as a rotation of the DDM horseshoe, as described earlier. Actually, what can be recognized as something similar to a rotation, consists of a delay of the waveforms (WFs) from the positive Doppler channels with respect to the WFs relating to those from the negative Doppler channels (or vice versa). Therefore, the test assesses the relative lag of the normalized $\mathrm{WF}$ at $1 \mathrm{kHz}$ with respect to the WF at $-1 k H z$. This test could also be implemented for other Doppler channels, but after some tests, the choosen channels provide the best compromise between the SNR and the sensitivity to the lag estimation. In order to appreciate lags smaller than $\tau_{c} / 4$ (delay grid spacing), both WFs have been oversampled by reducing the delay grid spacing to $\tau_{c} / 16$. The relative lag has been assessed in the interval $\left[-2.4375 \tau_{c}, 2.4375 \tau_{c}\right]$ that corresponds to \pm 39 bins in the new delay grid. Since WFs can be really noisy (figure 2c), a de-noising procedure based on Empirical Orthogonal Functions (EOFs) is applied. The EOF decomposition and the related Principal Component Analysis (PCA) are two powerful tools that can be used for several purposes, such as the dimensionality reduction in high computing demanding problems, the noise reduction and the linear regression in ill-posed problems [37]. Here, the de-noising procedure only aims at filtering some secondary peaks that can mask the real peak of the WF. The empirical basis is obtained through the singular value decomposition of the covariance matrix of the normalized WFs at $\pm 1 k H z$ for the entire DDM dataset, while the filtered normalized WFs is reconstructed by only using the first 3 principal components. Some sensitivity tests aiming at assessing the most appropriate number of components has been carried out, giving 3 as the optimal choice. The first 3 singular values explain respectively the $39 \%, 13 \%$ and $5 \%$ of the variance of the entire signal.

The two de-noised WFs are cross correlated with each other and $\arg \max _{k_{D}} P$ represents the lag, where $P$ is the cross correlation coefficient and $k_{D}$ the corresponding lag. $k_{D}=0$ represents the perfect alignment of the two WFs, while $k_{D}>0$ $\left(k_{D}<0\right)$ is related to an early arrival (delay) of the WF at $1 k H z$ with respect to the WF at $-1 k H z$. The equivalent relative shift $\Delta \tau_{D}$ is equal to $k_{D} \frac{\tau_{c}}{16}$. Here, the subscript $D$ stands for DATA and is used to distinguish this quantity from the geophysical effects, introduced below.

The main assumption of the $Q T_{2}$ is that both WFs at $\pm 1 \mathrm{kHz}$ arrive at the same time. Of course, this is not exactly true, but for incidence angles $<35^{\circ}$, the difference should be in the order of a fraction of a delay bin, as shown in figure 3 .

In figure 3, the red ellipse shows the instant of time at which the WF at $1 k H z$ reaches the receiver. As it can be seen, this 


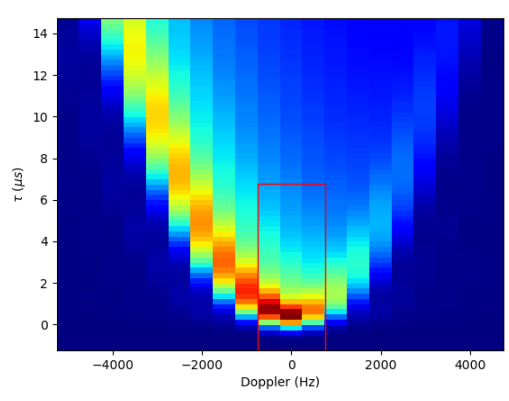

(a)

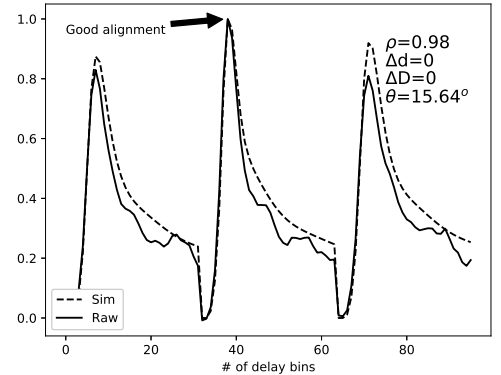

(b)

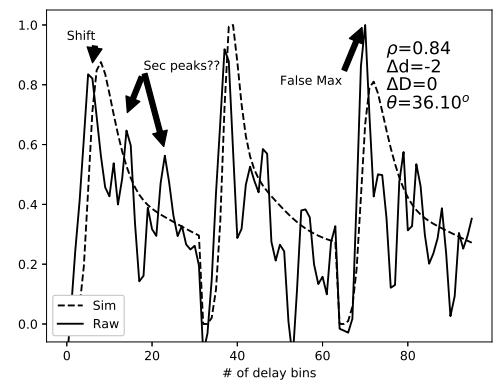

(c)

Fig. 2. In figure $2 \mathrm{a}$ an example of a simulated DDM is shown together with the inner core that is used for the delay/Doppler shift evaluation. Figures $2 \mathrm{~b}$ and $2 \mathrm{c}$ show two cases of 1D inner core DDMs. In both pictures, both the simulated (dotted line) and the real (solid line) DDMs are represented. In figure $2 \mathrm{~b}$ one can appreciate a good alignment and a very high SNR. In figure $2 \mathrm{c}$ one can see that the SNR is lower as well as a mis-alignment.

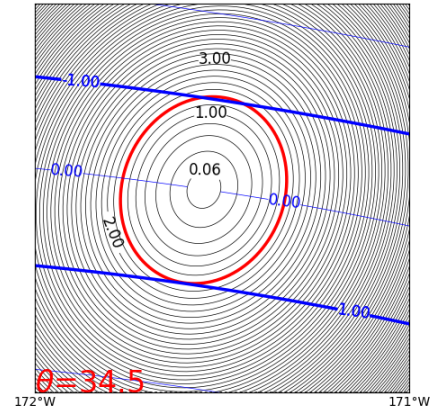

Fig. 3. Iso-delay (black solid lines) and iso-Doppler lines (blue solid lines) for an incidence angle $\theta \approx 35^{\circ}$. The red ellipse shows the iso-delay line approximately tangent to both iso-Doppler lines at $\pm 1 \mathrm{kHz}$ (solid bold blue lines). This instant of time $\left(1.5 \tau_{c}\right)$ represents the arrival time of the WFs at $\pm 1 k H z$. It can be seen that the WF at $-1 k H z$ arrives slightly later than the WF at $1 k H z$, but this difference is only a fraction of a delay bin.

instant of time is almost equal to the arrival time of the WF at $-1 \mathrm{kHz}$, the difference of which being a small fraction of a delay bin. It follows that a higher difference in the arrival time of the two WFs can be due to an incorrect estimation of the SP with a consequent alteration of the symmetry between isodelay and iso-Doppler lines. In some special cases, the test can give a non null lag estimation even if the arrival time of the WFs is almost identical. This can happen when there is high wind speed variability. Indeed, in such conditions, the WF of the $1 \mathrm{kHz}$ channel can peak in a quite different position with respect to the $\mathrm{WF}$ at $-1 k H z$, giving rise to a non null lag. This effect is not to be attributed to any distortion, as it is rather a "geophysical" effect. This shift can be computed by applying the same cross correlation procedure to the synthetic WFs, and can be taken into account by algebraically subtracting it from $\Delta \tau_{D}$ as follows:

$$
\Delta \tau=\Delta \tau_{D}-\Delta \tau_{G}
$$

where the subscript $G$ stands for geophysical and $\Delta \tau$ is the final output of the test $Q T_{2}$.

In principle, also the antenna gain pattern, the residual speckle noise and thermal noise could lead to some asymmetries of the DDM, however, this paper demonstrates that the main cause of such asymmetries is the SP estimation mismatch.

It is important to stress that the same quality control scheme has also been adapted to regular CYGNSS-like DDMs that are operationally delivered in the $17 \times 11$ delay-Doppler format. Indeed, such DDMs give information in the Doppler range between $-2.5 \mathrm{kHz}$ and $2.5 \mathrm{kHz}$ and the delay range is between $-1 \tau_{c}$ and $3 \tau_{c}$, the grid sampling spaces being identical to those of the TDS-1 DDMs. For such purpose, the $Q T_{1}$ is computed in an inner DDMs core of $3 \times 17$ bins (same Doppler range and the entire CYGNSS delay range) and the $Q T_{2}$ is applied to the same WFs at $\pm 1 k H z$ for the entire CYGNSS delay range. In the following section, only the results of the quality control scheme applied to the TDS-1 dataset are presented for the sake of brevity, while we note that the results on the CYGNSS-like DDMs are similar to those of the full TDS-1 DDMs, and only slightly degraded because of the reduced number of DDM bins.

\section{RESULTS AND DISCUSSION}

\section{A. Quality control test 1}

Figure 4 shows an example of the application of the quality control scheme. Figure $4 \mathrm{~b}$ shows a DDM as it is acquired by the SGR-ReSI instrument. From a visual inspection, one can note a positive delay shift with respect to the simulated one, which is depicted in figure 4a. After the application of the $Q T_{1}$, the DDM is repositioned as depicted in figure 4c. Now, it better overlaps with the simulated DDM. At the same time, one can also appreciate a delay of the WFs in the negative Doppler channels with respect to those in the positive channels. This can be seen more clearly in figure $4 \mathrm{~d}$, where the normalized WFs at $1 \mathrm{kHz}$ (solid red line) and at $-1 k H z$ (solid blue line) are depicted. It is evident that the $\mathrm{WF}$ at $1 k H z$ arrives earlier than the WF at $-1 k H z$. One can compare the two normalized WFs with those simulated that are depicted respectively with dotted red and blue lines. It is hard to distinguish the simulated WFs at $\pm 1 \mathrm{kHz}$ because they almost perfectly overlap. Therefore, the quantity $\Delta \tau_{G}$ is negligible here and the $\Delta \tau$ is mainly due to a distortion effect. One can also note that the shift does not only affect the peaks of the WFs, but also the leading and trailing edges, confirming the presence of some distortion. 


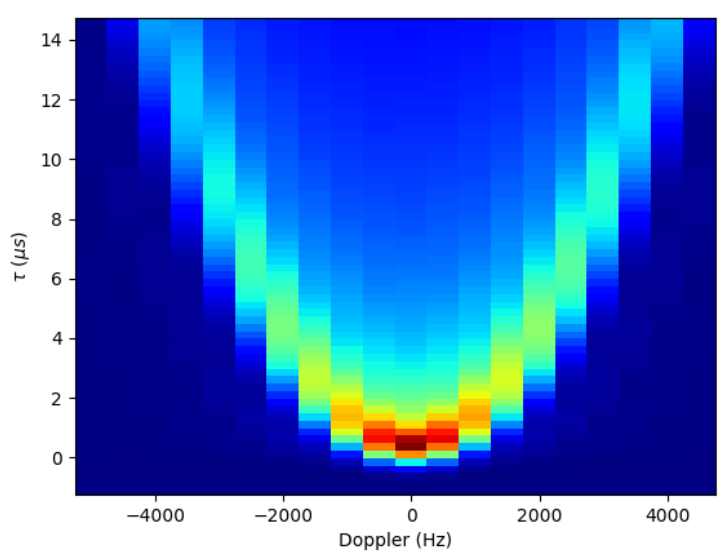

(a)

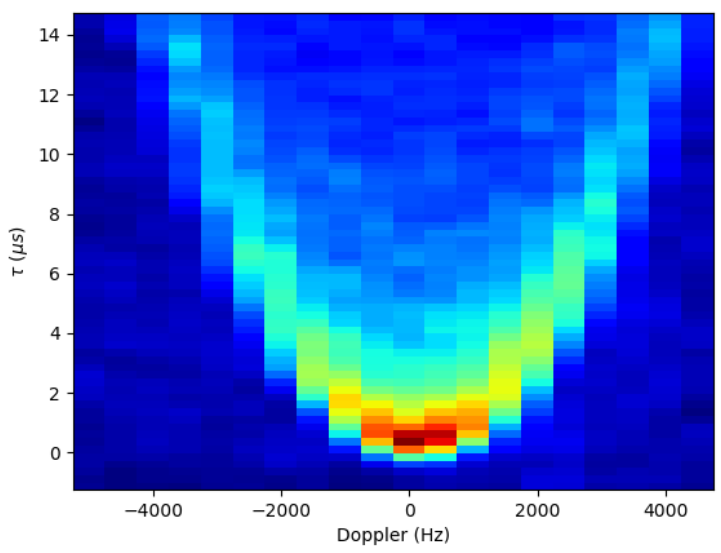

(c)

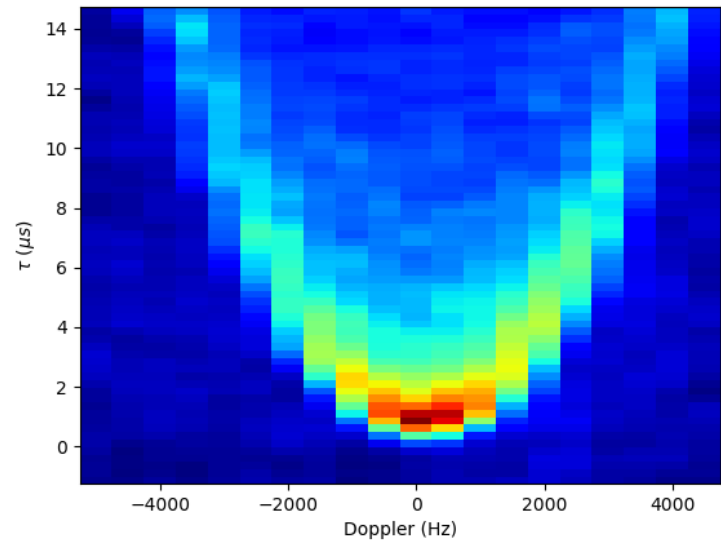

(b)

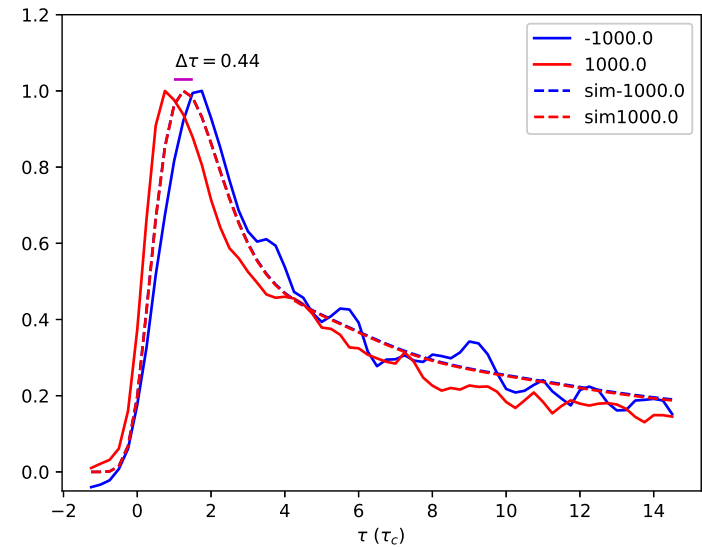

(d)

Fig. 4. Example of the application of the quality control scheme. Figure 4a shows the simulated DDM, figure 4b is the raw DDM as acquired by SGR-ReSI, while figure $4 \mathrm{c}$ is the result of the repositioning after $Q T_{1}$ is applied. Finally, figure $4 \mathrm{~d}$ shows the normalized WFs of the acquired DDM (after repositioning) at $\pm 1 \mathrm{kHz}$ respectively in solid red and solid blue lines. Simulated WFs at $\pm 1 \mathrm{kHz}$ are indicated by the dotted lines (respectively red and blue). It can be seen that the arrival time of the real WFs is different, giving the perception of a rotation of the DDM horseshoe.

Figure 5 shows the distribution of the cross correlation $(\rho)$ coefficient from $Q T_{1}$. In the panel legend, a few statistical parameters about the distribution are shown, such as: the $10^{\text {th }}$ percentile, the median and the maximum $\rho$. The total number of DDMs is slightly lower than that of the original dataset because in some cases, $Q T_{1}$ cannot be applied due to some missing numbers in the raw DDM. These occurrences represent a small percentage $(\approx 1 \%)$ of the whole dataset and are properly stated in the metadata.

Note the contrast between the DDMs with high value of $\rho$ (see figure 2) and those with small $\rho$ (figure 6).

Figure 6 shows two DDMs that are rejected by $Q T_{1}$. Figure 6a shows some artifacts that are probably due to problems in the data acquisition and/or processing. Indeed, the WFs at $0.5 \mathrm{kHz}$ and $1 \mathrm{kHz}$ show a much higher intensity with respect to the surrounding WFs. Furthermore, there is only a slight trace of the horseshoe in the area surrounding the DDM peak bin. It is important to stress that this DDM is not flagged in the metadata while $Q T_{1}$ has been able to do it.
Figure $6 \mathrm{~b}$ depicts a noisy DDM, where some secondary peaks, such as those shown in the 1D DDM of figure 2c, can be easily recognized in most of the WFs, especially those between $0 \mathrm{kHz}$ and $2 \mathrm{kHz}$. If all the DDMs shown in figures 2 and 6 are sorted according to the $\rho$ values, one can recognize that they are in perfect agreement with a visual check. For the remaining of this analysis, only DDMs with $\rho>0.9$ have been considered.

In order to have a synoptic view of the effectiveness of this quality control criterion, figures 7 a to $7 \mathrm{c}$ show a $2 \mathrm{D}$ histogram of the ASCAT wind speed versus the SNR for Range Corrected Gain (RCG) > 3 (currently used for CYGNSS data, 7a), RCG $>11.56(7 \mathrm{~b})$ and $\rho>0.9(7 \mathrm{c})$. These histograms refer to all TDS-1/ASCAT collocated data with TDS- 1 incidence angle at SP $<45^{\circ}$. The total number of samples amounts to about 110,000 . When we apply the criterion $\rho>0.9$, the number of samples reduces to about 78,400 samples, that is considerably lower than that of RCG $>3$. Therefore, the criterion RCG $>11.56$ has been considered in order to have a similar number 


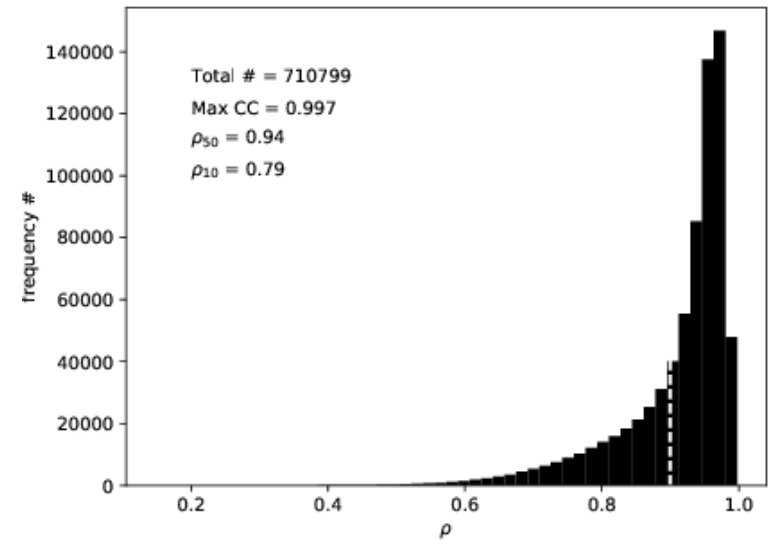

Fig. 5. Distribution of the cross correlation coefficient $(\rho)$ from $Q T_{1}$. The legend shows some statistics about the distribution, such as the $10^{\text {th }}$ percentile $\left(\rho_{10}\right)$, the median $\left(\rho_{50}\right)$ and the maximum value of $\rho$. Finally, the white dotted line represents the minimum threshold value that flags good quality DDMs: $\rho=0.9$.

of samples to those obtained with $\rho>0.9$. Figure $7 \mathrm{~d}$ shows the standard deviation of SNR associated to all criteria versus ASCAT wind speed in the range $0-20 \mathrm{~ms}^{-1}$. For the sake of completeness, the standard deviation before any quality control is applied, is reported and stated with "No QC".

From figure $7 \mathrm{~d}$ it can be seen that the standard deviation of SNR significantly reduces when $\rho>0.9$ is applied with respect to all other criteria. Most of the SD reduction is in the wind speed range $0-7 \mathrm{~ms}^{-1}$, while the SD reduction is less noticeable for winds above $7 \mathrm{~ms}^{-1}$ (when compared against RCG > 11.56). We have verified that the collocated ASCAT wind speed distribution is similar to that of the real global wind speed distribution. As such, one can conclude that a significant SD reduction is achieved for the proposed QC filter for about half of the data points (i.e., winds lower than $\left.7 \mathrm{~ms}^{-1}\right)$. In addition, it seems that most of the outliers in the upper part of the scatter plot in Figures $7 \mathrm{a}$ and $7 \mathrm{~b}$ are effectively removed when the quality control criterion $\rho>0.9$ is applied in Figure 7c.

\section{B. Quality control test 2}

The analysis has been limited to the scenes that have been acquired with an incidence angle lower than $35^{\circ}$ at the SP. In this way, one is sure that the symmetry between iso-delay and iso-Doppler lines is preserved with a high accuracy (see figure 3). This criterion, together with the constraint $\rho>0.9$ limits the number of samples to $63 \%$ of the total amount of data. Figure 8 shows the distribution of the two components of the relative delay shift of the WFs at $\pm 1 k H z: \Delta \tau_{D}$ and $\Delta \tau_{G}$, respectively in blue and in yellow.

It can be seen that the two distributions are quite different. The distribution of $\Delta \tau_{G}$ is much narrower than that of $\Delta \tau_{D}$, stressing that the geophysical component of $\Delta \tau$ plays a minor role. Furthermore, while the distribution of $\Delta \tau_{G}$ is approximately Gaussian and centered at 0 , the one of $\Delta \tau_{D}$ is rather bimodal, asymmetric and with an expectation value different than 0 . The mean value of $\Delta \tau_{D}$ is $0.16 \tau_{c}$, more than half a delay bin. The cross correlation of $\Delta \tau_{G}$ and $\Delta \tau_{D}$ is almost 0 , stressing once more that the nature of $\Delta \tau_{D}$ is not related to the wind at the observed scene.

In order to prove that $\Delta \tau_{D}$ is mainly caused by an inaccurate estimation of the SP location, this parameter has been correlated to the Doppler component of the vector difference between the SP location computed through WAVPY and the SP location provided in the metadata [28]. WAVPY an iterative Newton-Raphson method that builds a gradient function by applying three conditions: (1) location over the WGS84 ellipsoid; (2) fulfillment of Snell's law; and (3) co-planarity of specularto-receiver, specular-to-transmitter and surface-normal vectors. Therefore, it has been assumed as the reference truth. This parameter ( $\Delta f_{0}$, where 0 stands for 0 -Doppler) has been computed according to the following equation:

$$
\Delta f_{0}=f_{W P}-f_{T D S-1} \approx \frac{\mathbf{V}_{\mathbf{R}} * \mathbf{m}^{\mathbf{T D S}-\mathbf{1}}}{\lambda}-\frac{\mathbf{V}_{\mathbf{R}} * \mathbf{m}^{\mathbf{W P}}}{\lambda}
$$

In equation 3, $f_{W P}$ and $f_{T D S-1}$ are the Doppler shift of the carrier frequency caused by the relative motion of both the receiver (subscript $R$ ) and the transmitter evaluated by means of the SP location computed with WAVPY (subscript WP) and of the SP location provided by the TDS-1 metadata (subscript TDS-1). $\mathbf{m}$ represents the outgoing unitary vector at the SP towards the receiver. This formula is approximated because the component of the Doppler shift due to the transmitter velocity is quite similar for both SP locations, therefore they cancel out. In other words, this is the Doppler difference between the 0 -Doppler reference obtained with the reference SP location and the 0-Doppler reference obtained with the SP location provided in the metadata.

Figure 9 shows the scatter plot of $\Delta f_{0}$ and $\Delta \tau$ with some additional information in the legend, such as the Pierson correlation coefficient (r), the slope and the intercept of the fitting curve ( $\mathrm{a}$ and $\mathrm{b}$, respectively) and the probability associated to the null hypothesis test (p). The fitting curve is depicted with a dotted magenta line. The color bar is in logarithmic scale in order to magnify the patterns of the scatter plot. In figure 9 one can recognize the same features of the distribution of $\Delta \tau_{D}$. Indeed, the two peaks of the distribution are depicted in the two red spots of the scatter plot. The high correlation of $\Delta \tau$ with $\Delta f_{0}$ proves that the SP inaccuracy is the main cause of this feature. Figure 8 shows that the Pierson correlation coefficient between $\Delta \tau$ and $\Delta f_{0}$ is slightly higher than that between $\Delta \tau_{D}$ and $\Delta f_{0}$, in line with what is expected. Also the intercept of the fitting curve is in line with the expected value of $\Delta \tau$, even if a little bit higher. Furthermore, $\Delta f_{0}$ is approximately in the range $[-220,220] \mathrm{Hz}$ in agreement with what is stated in [28].

Finally, no evidence of the effects of the antenna gain pattern, of the residual speckle noise and thermal noise on DDM asymmetry has been reported during this analysis. This is not to deny their effects, but it is evident that they are of second order with respect to the effects induced by an inaccurate estimation of the SP location. 


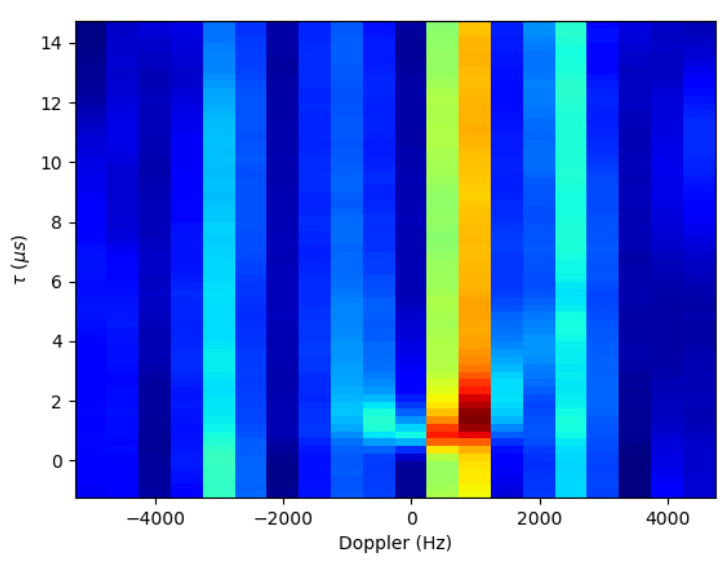

(a)

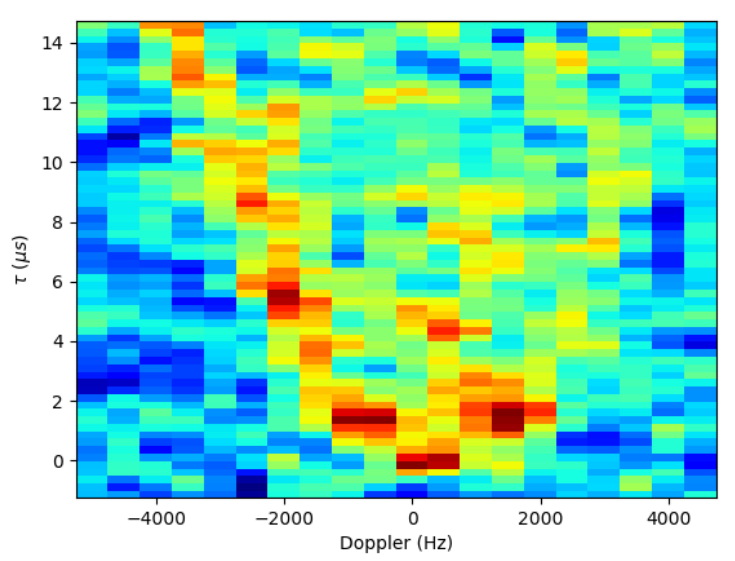

(b)

Fig. 6. Example of two cases that are rejected by $Q T_{1}$. Figure 6a evidences two more intense WFs at $0.5 k H z$ and $1 k H z$ with respect to the other WFs. Furthermore, there is only a slight trace of the horseshoe. $6 \mathrm{~b}$ shows a really noisy DDM, where the secondary peaks shown in figure $2 \mathrm{c}$ can be easily recognized in the trailing edges of the WFs, especially those between $0 \mathrm{kHz}$ and $2 \mathrm{kHz}$.

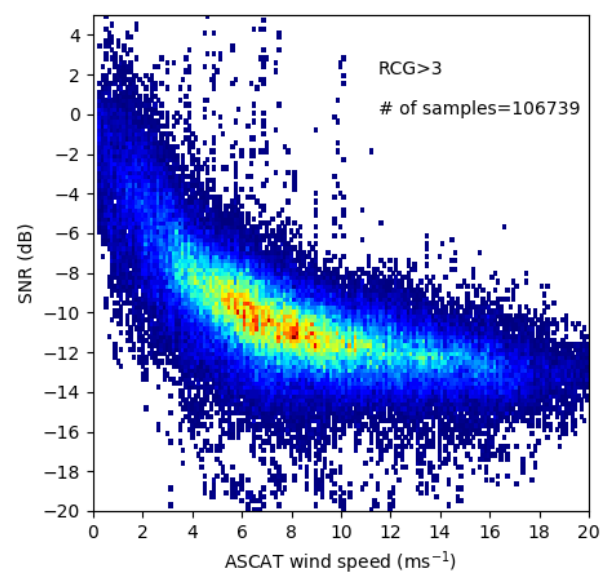

(a)

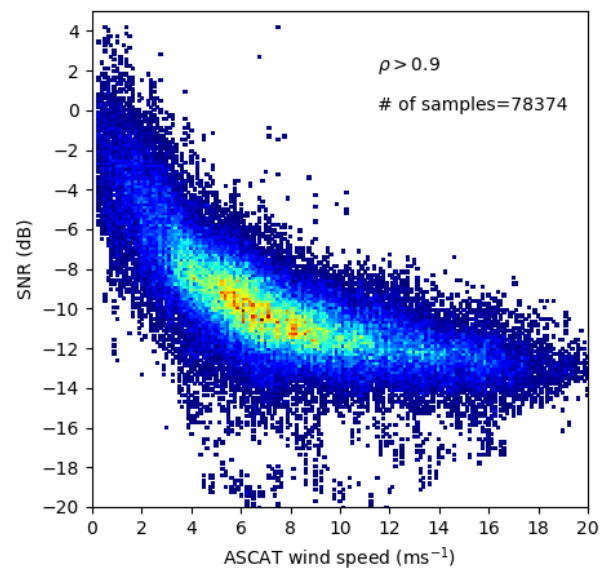

(c)

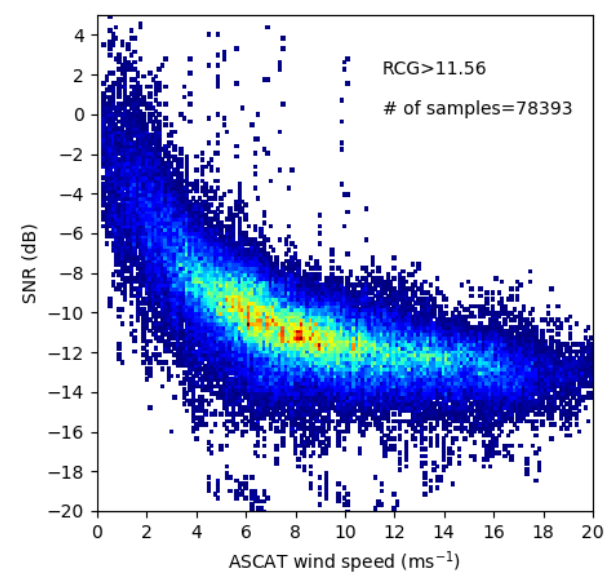

(b)

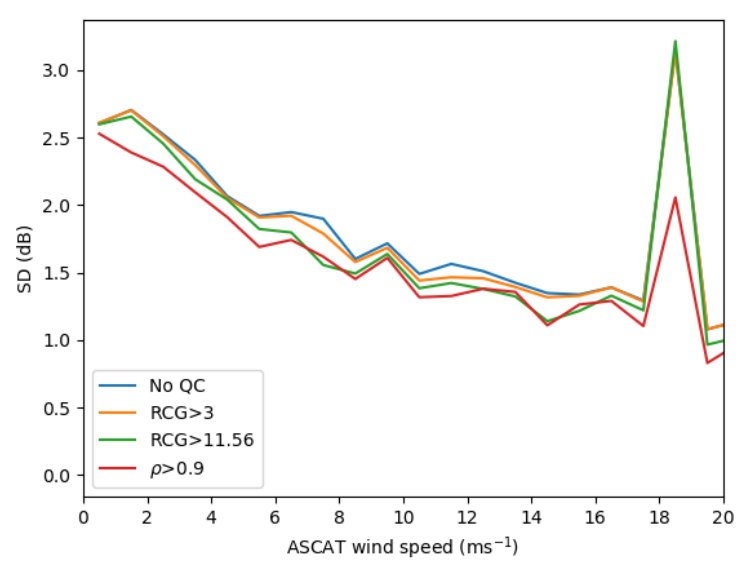

(d)

Fig. 7. Density plot of ASCAT wind speed versus TDS-1 SNR (a) to c)) and standard deviation of SNR with respect to ASCAT wind speed (d)). The number of samples that are selected by each criterion is reported in the legend. "No QC" in 7d only refers to collocation criteria. 


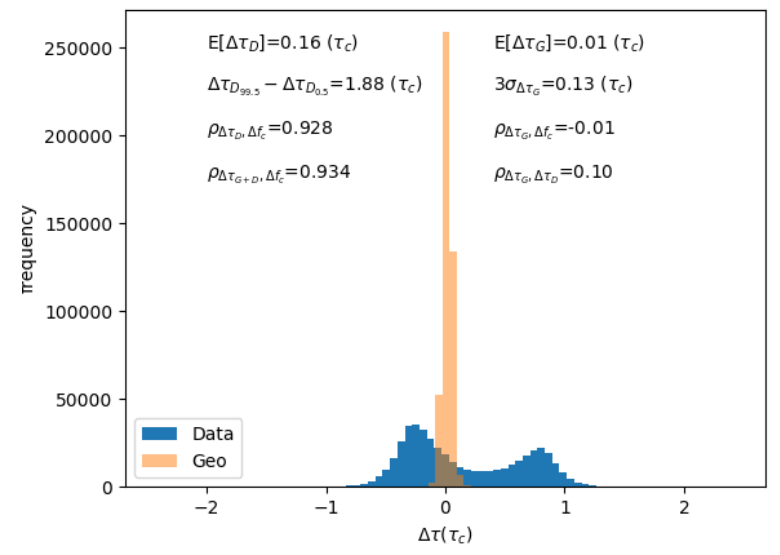

Fig. 8. Distribution of the two components of $\Delta \tau$ from $Q T_{2}$. The distribution of $\Delta \tau_{D}$ is depicted in blue, while that of $\Delta \tau_{G}$ is in yellow. Some additional information is provided in the legend: the expected value, the $99 \%$ confidence interval, the mutual cross correlation coefficient, the cross correlation coefficient of both $\Delta \tau_{D}$ and $\Delta \tau_{G}$ with $\Delta f_{0}$ and the cross correlation coefficient of $\Delta \tau$ with $\Delta f_{0}$

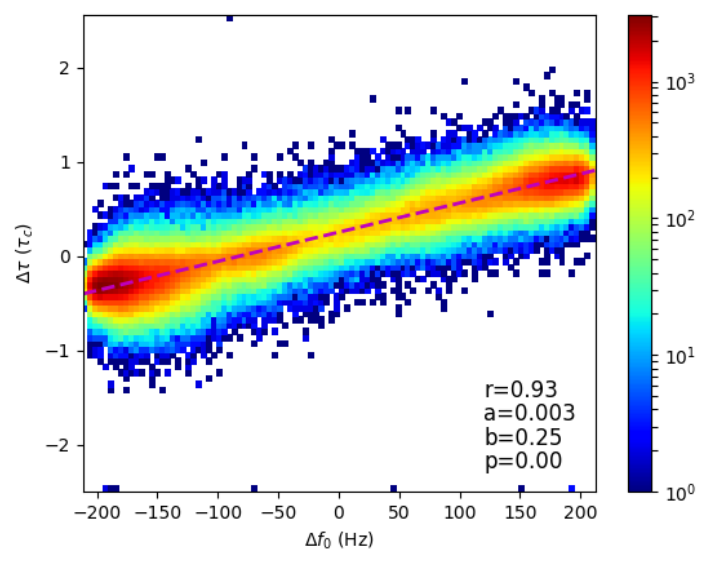

Fig. 9. Scatter plot of the relative shift between the WFs at $\pm 1 k H z(\Delta \tau)$ and the Doppler difference between the 0-Doppler computed with the reference SP and the 0-Doppler computed with the SP provided in the TDS-1 metadata $\left(\Delta f_{0}\right) . \mathrm{r}$ is the Pierson correlation coefficient, a is the slope, b is the intercept of the fitting curve (dotted magenta line), and $\mathrm{p}$ is the probability associated to the null hypothesis test.

\section{CONCLUSIONS AND FUTURE WORK}

A quality control scheme based on the cross correlation function is designed for both TDS-1 and regular CYGNSS DDMs. This quality control scheme is applied to a dataset of more than 700,000 TDS-1 DDMs and the results have been discussed. The output indices of the tests can be successfully used as quality indicators of the DDM, assessing a performance that is significantly better than the Range Gain Corrected criterion operationally implemented for CYGNSS DDM.

The quality control scheme proposed in this paper has proven to be effective for diagnosing TDS-1 DDM distortions, while giving similar results for the regular CYGNSS DDM format. Most of the TDS-1 DDMs are affected by distortions that are attributable to an inaccurate estimation of the specular point (SP) location. An inaccurate estimation of the SP location may give rise to multiple artifacts, such as a) lagging of the echo, b) the alteration of the symmetry between delay and Doppler iso-lines, c) a redistribution of the echo energy in the DDM bins due to a mis-alignment of the echo with the WAF. While an a posteriori re-computation of the SP can improve the estimation of the arrival of the echo [28], this information cannot be used to reduce the effects of DDM distortion described in points b) and c). Such effects may be corrected only by reprocessing the raw data, but this is not possible as long as L0 data are not available. For such reason, it is highly recommended that L0 will be made available in the near future. The analyzed distortions may compromise the application of the stare processing approach [27], but also severely affect the more consolidated procedures of retrieving the wind speed through a GMF-based approach on the DDM peak and/or the leading edge slope (LES) [12], [20]-[22]. Indeed, it is not yet assessed how much the effects described in point c) above can affect the DDM peak intensity and the LES. Such topic goes beyond the scope of this paper, but it is highly recommended that some ad-hoc sensitivity studies are carried out in the near future.

\section{ACKNOWLEDGMENT}

This work has been carried out in the context of the EUMETSAT fellowship project GOODIE: GNSS-R Observation Operator Development and Impact Evaluation.

\section{REFERENCES}

[1] C. Cardinali, "Monitoring the observation impact on the shortrange forecast," Quarterly Journal of the Royal Meteorological Society, vol. 135 , no. 638, pp. 239-250, 2009. [Online]. Available: https://rmets.onlinelibrary.wiley.com/doi/abs/10.1002/qj.366

[2] (2017) ECV Atmosphere Matrix. [Online]. Available: https://public.wmo.int/en/programmes/global-climate-observingsystem/essential-climate-variables

[3] A. Stoffelen, A. Verhoef, J. Verspeek, J. Vogelzang, G. Marseille, T. Driesenaar, Y. Risheng, G. D. Chiara, C. Payan, J. Cotton, A. Bentamy, and M. Portabella, "Research and development in europe on global application of the oceansat-2 scatterometer winds," KNMI-NWP SAF-OSI SAF, MA, Tech. Rep. NWPSAF-KNTR-022-SAF/OSI/CDOP2/KNMI/TEC/RP/196, May 2013. [Online]. Available: https://www.nwpsaf.eu/publications/tech_reports/nwpsaf-kntr-022.pdf?e6316e\&e6316e

[4] A. Stoffelen, J. A. Verspeek, J. Vogelzang, and A. Verhoef, "The cmod7 geophysical model function for ascat and ers wind retrievals," IEEE Journal of Selected Topics in Applied Earth Observations and Remote Sensing, vol. 10, no. 5, pp. 2123-2134, May 2017.

[5] J. Vogelzang, A. Stoffelen, R. D. Lindsley, A. Verhoef, and J. Verspeek, "The ascat 6.25-km wind product," IEEE Journal of Selected Topics in Applied Earth Observations and Remote Sensing, vol. 10, no. 5, pp. 2321-2331, May 2017.

[6] J. Zhu, X. Dong, W. Lin, and D. Zhu, "A preliminary study of the calibration for the rotating fan-beam scatterometer on cfosat," IEEE Journal of Selected Topics in Applied Earth Observations and Remote Sensing, vol. 8, no. 2, pp. 460-470, Feb 2015.

[7] (2017) Wmo oscar database. [Online]. Available: https://www.wmosat.info/oscar/

[8] Wmo oscar database. [Online]. Available: http://ceos.org/ourwork/virtual-constellations/osvw/

[9] (2017) The GOODIE webpage. [Online]. Available: http://www.knmi.nl/kennis-en-datacentrum/project/global-navigationsatellite-system-observation-operator-development-and-impactevaluation-goodie 
[10] E. Cardellach, F. Fabra, O. NogusCorreig, S. Oliveras, S. Rib, and A. Rius, "Gnssr groundbased and airborne campaigns for ocean, land, ice, and snow techniques: Application to the goldrtr data sets," Radio Science, vol. 46, no. 6, 2011. [Online]. Available: https://agupubs.onlinelibrary.wiley.com/doi/abs/10.1029/2011RS004683

[11] E. Cardellach, G. Ruffini, D. Pino, A. Rius, A. Komjathy, and J. L. Garrison, "Mediterranean balloon experiment: ocean wind speed sensing from the stratosphere, using gps reflections," Remote Sensing of Environment, vol. 88, no. 3, pp. 351 - 362, 2003. [Online]. Available: http://www.sciencedirect.com/science/article/pii/S0034425703001767

[12] G. Foti, C. Gommenginger, P. Jales, M. Unwin, A. Shaw, C. Robertson, and J. Rosell, "Spaceborne gnss reflectometry for ocean winds: First results from the uk techdemosat1 mission," Geophysical Research Letters, vol. 42, no. 13, pp. 5435-5441, 2015. [Online]. Available: https://agupubs.onlinelibrary.wiley.com/doi/abs/10.1002/2015GL064204

[13] J. L. Garrison and S. J. Katzberg, "Detection of ocean reflected gps signals: theory and experiment," in Southeastcon '97. Engineering new New Century., Proceedings. IEEE, Apr 1997, pp. 290-294.

[14] J. L. Garrison, S. J. Katzberg, and M. I. Hill, "Effect of sea roughness on bistatically scattered range coded signals from the global positioning system," Geophysical Research Letters, vol. 25, no. 13, pp. 2257-2260, 1998. [Online]. Available: https://agupubs.onlinelibrary.wiley.com/doi/abs/10.1029/98GL51615

[15] V. U. Zavorotny and A. G. Voronovich, "Scattering of gps signals from the ocean with wind remote sensing application," IEEE Transactions on Geoscience and Remote Sensing, vol. 38, no. 2, pp. 951-964, Mar 2000.

[16] A. G. Voronovich and V. U. Zavorotny, "The transition from weak to strong diffuse radar bistatic scattering from rough ocean surface," IEEE Transactions on Antennas and Propagation, vol. 65, no. 11, pp. 60296034, Nov 2017.

[17] _ _Bistatic radar equation for signals of opportunity revisited," IEEE Transactions on Geoscience and Remote Sensing, vol. 56, no. 4, pp. 1959-1968, April 2018.

[18] M. Unwin, P. Jales, J. Tye, C. Gommenginger, G. Foti, and J. Rosello, "Spaceborne gnss-reflectometry on techdemosat-1: Early mission operations and exploitation," IEEE Journal of Selected Topics in Applied Earth Observations and Remote Sensing, vol. 9, no. 10, pp. 4525-4539, Oct 2016.

[19] C. S. Ruf, R. Atlas, P. S. Chang, M. P. Clarizia, J. L. Garrison, S. Gleason, S. J. Katzberg, Z. Jelenak, J. T. Johnson, S. J. Majumdar, A. Obrien, D. J. Posselt, A. J. Ridley, R. J. Rose, and V. U. Zavorotny, "New ocean winds satellite mission to probe hurricanes and tropical convection," Bulletin of the American Meteorological Society, vol. 97, no. 3, pp. 385-395, 2016. [Online]. Available: https://doi.org/10.1175/BAMS-D-14-00218.1

[20] S. Soisuvarn, Z. Jelenak, F. Said, P. S. Chang, and A. Egido, "The gnss reflectometry response to the ocean surface winds and waves," IEEE Journal of Selected Topics in Applied Earth Observations and Remote Sensing, vol. 9, no. 10, pp. 4678-4699, Oct 2016.

[21] W. Lin, M. Portabella, G. Foti, A. Stoffelen, C. Gommenginger, and Y. He, "Towards the generation of a wind geophysical model function for space-borne gnss-r," IEEE Transactions on Geoscience Remote Sensing, 2018 , in press.

[22] M. P. Clarizia, C. S. Ruf, P. Jales, and C. Gommenginger, "Spaceborne gnss-r minimum variance wind speed estimator," IEEE Transactions on Geoscience and Remote Sensing, vol. 52, no. 11, pp. 6829-6843, Nov 2014.

[23] N. Rodriguez-Alvarez and J. L. Garrison, "Generalized linear observables for ocean wind retrieval from calibrated gnss-r delay doppler maps," IEEE Transactions on Geoscience and Remote Sensing, vol. 54, no. 2 , pp. $1142-1155$, Feb 2016.

[24] C. S. Ruf, S. Gleason, and D. S. McKague, "Assessment of cygnss wind speed retrieval uncertainty," IEEE Journal of Selected Topics in Applied Earth Observations and Remote Sensing, pp. 1-11, 2018.

[25] A. Verhoef, J. Vogelzang, J. Verspeek, and A. Stoffelen, "Long-term scatterometer wind climate data records," IEEE Journal of Selected Topics in Applied Earth Observations and Remote Sensing, vol. 10, no. 5, pp. 2186-2194, May 2017.

[26] G. Foti, C. Gommenginger, M. Unwin, P. Jales, J. Tye, and J. Rosell, “An assessment of non-geophysical effects in spaceborne gnss reflectometry data from the uk techdemosat-1 mission," IEEE Journal of Selected Topics in Applied Earth Observations and Remote Sensing, vol. 10, no. 7, pp. 3418-3429, July 2017.

[27] J. Tye, P. Jales, M. Unwin, and C. Underwood, "The first application of stare processing to retrieve mean square slope using the sgr-resi gnss-r experiment on tds-1," IEEE Journal of Selected Topics in Applied Earth
Observations and Remote Sensing, vol. 9, no. 10, pp. 4669-4677, Oct 2016.

[28] MERRByS Product Manual - GNSS Reflectometry on TDS-1 with the SGR-ReSI Version 4, Surret Satellite Technology Limited, 2018.

[29] P. Jales, "Spaceborne receiver design for scatterometric gnss reflectometry," Ph.D. dissertation, Univ. of Surrey, 2012. [Online]. Available: http://epubs.surrey.ac.uk/807880/

[30] S. Gleason, C. S. Ruf, M. P. Clarizia, and A. J. O'Brien, "Calibration and unwrapping of the normalized scattering cross section for the cyclone global navigation satellite system," IEEE Transactions on Geoscience and Remote Sensing, vol. 54, no. 5, pp. 2495-2509, May 2016.

[31] P. Steigenberger, S. Thoelert, and O. Montenbruck, "Gnss satellite transmit power and its impact on orbit determination," Journal of Geodesy, vol. 92, no. 6, pp. 609-624, Jun 2018. [Online]. Available: https://doi.org/10.1007/s00190-017-1082-2

[32] S. Thoelert, M. Meurer, and S. Erker, "In-orbit analysis of antenna pattern anomalies of gnss satellites," Navigation, vol. 59, no. 2, pp. 135-144. [Online]. Available: https://onlinelibrary.wiley.com/doi/abs/10.1002/navi.11

[33] F. Fabra, E. Cardellach, W. Li, and A. Rius, "Wavpy: A gnss-r open source software library for data analysis and simulation," in 2017 IEEE International Geoscience and Remote Sensing Symposium (IGARSS), July 2017, pp. 4125-4128.

[34] S. J. Katzberg, O. Torres, and G. Ganoe, "Calibration of reflected gps for tropical storm wind speed retrievals," Geophysical Research Letters, vol. 33, no. 18, 2006. [Online]. Available: https://agupubs.onlinelibrary.wiley.com/doi/abs/10.1029/2006GL026825

[35] wavpy TGSCatt v4.3: User manual, Institute of Space Studies of Catalonia, 2017.

[36] CYGNSS Handbook Cyclone Global Navigation Satellite System. Deriving Surface Wind Speeds in Tropical Cyclones, University of Michigan, 2016. [Online]. Available: http://www.publishing.umich.edu/services/

[37] G. G. C. Serio, G. Masiello, Environmental Modelling: New Research. NOVA Science Publisher Inc., 2008, ch. 2.

\begin{tabular}{|c|}
\hline \\
\\
PLACE \\
PHOTO \\
HERE \\
\end{tabular}

Giuseppe Grieco Giuseppe Grieco received his M. Sc. in Marine Sciences from the University Parthenope of Napoli in 2001 and his Ph. D. in Environmental Engineering at the Universit della Basilicata in 2008. Currently, he has a EUMETSAT fellowship on GNSS-Reflectometry that has being carrying out at KNMI in collaboration with the Institute of Marine Sciences of Barcelona (Spain).

Ad Stoffelen Ad Stoffelen (M'13-SM'14) received his M.Sc. in Physics from the Technical University of Eindhoven in 1987 and his Ph.D in Meteorology on Scatterometry at the University of Utrecht in 1998. Currently, he leads a group on active satellite sensing at KNMI and is involved in topics ranging from future missions and $R \& D$ for retrieval to 24/7 operations, NWP mesoscale wind data assimilation, user training and services. Establishing an international scatterometer virtual constellation is one of his main aims. He is also deeply involved in the ESA ADM-Aeolus Doppler Wind Lidar mission.

Marcos Portabella Marcos Portabella was born on October 14, 1970, in Spain. He received the B.Sc. degree in physics from the University of Barcelona, Barcelona, Spain, in 1994, the M.Sc. degree in remote sensing from the Institute of Space Studies of Catalonia, Barcelona, Spain, in 1995, and the Ph.D. degree in physics from the University of Barcelona, Spain, in 2002. He is currently with the Institute of Marine Sciences, Barcelona, Spain, working on satellite remote sensing. In particular, he is involved in scatterometry and L-band radiometry. 
Maria Belmonte Rivas Maria Belmonte Rivas received the B.Sc. degree in earth sciences from the Universidad Complutense de Madrid, Madrid, Spain, in 1999, and the M.Sc. and Ph.D. degrees in physics and aerospace engineering from the University of Colorado in Boulder, Boulder, CO, USA, in 2007. She is interested in the development of remote sensing techniques for Earth observation. She has held various positions at the European Space Research and Technology Centre, European Space Agency, Noordwijk, The Netherlands; the Colorado Center for Astrodynamics Research, University of Colorado in Boulder; the Atmospheric Chemistry Division, National Center for Atmospheric Research, Boulder, and the Technical University of Delft, Delft, The Netherlands. She is currently with the Royal Netherlands Meteorological Institute, De Bilt, The Netherlands.

Wenming Lin Wenming Lin (M16 SM 16) was born on April 22, 1984 in China. He received his B.Sc. degree in Engineering in 2006 from Wuhan University, China, and his Ph.D. in Engineering in 2011 at the National Space Science Center, Chinese Academy of Sciences. From 2011 to 2017, he was a postdoctoral researcher at the Institut de Cincies del Mar (ICM CSIC) in Barcelona, Spain, working on the advanced oceanographic data processing methods, remote sensing of ocean surface winds and data assimilation. He is currently a faculty member at Nanjing University of Information Science and Technology, working on ocean remote sensing.

Fran Fabra Dr. Fran Fabra received the M.Sc. degree in telecommunication engineering, in 2007, Masters degree in information and communication technologies from Universitat Politecnica de Catalunya (UPC), in 2007, and the $\mathrm{Ph} . \mathrm{D}$. degree from the Universitat Politecnica de Catalunya, Barcelona, Spain, in 2013. He developed his Masters thesis on security in aeronautical mobile networks at TriaGnoSys GmbH, Munich, Germany. His Ph.D. dissertation was dedicated to research of the GNSS-R concept toward remote sensing of the cryosphere. He is with the Earth Observation Research Group, Institute of Space Sciences (ICE-CSIC/IEEC), Barcelona, Spain, where he works on applications of global navigation satellite systems to Earth science. He is the main developer of the open source wavpy library for GNSS-R analysis and modelling. 